\title{
The serum level of cystatin $C$ does not predict dopaminergic cell death in substantia nigra, evidence from 6-hydroxydopamine-induced animal model of Parkinson's disease
}

\author{
Ali Sarbazi Golezari, Hashem Haghdoost-Yazdi*iD, Azita Minaei \\ Cellular and Molecular Research Center, Research Institute for Prevention of Non- Communicable Disease, Qazvin \\ University of Medical Sciences, Qazvin, Iran
}

\begin{abstract}
Introduction: Cystatin C (CysC) is an indicator of renal function, and has been recently reported that associates with neurodegenerative diseases. To investigate the role of this substance in Parkinson's disease (PD), we evaluated the association between serum levels of CysC and other markers of renal function with 6hydroxydopamine (6-OHDA) induced Parkinsonism in rat.
\end{abstract}

Methods: The 6-OHDA was microinjected into the medial forebrain bundle by stereotaxic surgery. After behavioral tests, immunofluorescence and biochemical studies were carried out to determine the number of survived dopaminergic (DA) neurons in the substantia nigra pars compacta (SNc) and striatal dopamine level. The blood samples were collected from the caudal vain and the heart of animals. CysC was measured using the enzyme-linked immunosorbent assay (ELISA) kits.

Results: There was no difference in serum level of CysC between the 6-OHDA treated and control groups, as well as before and after the toxin in the 6-OHDA group. Also, no association was found between CysC and DA neuronal death in SNc or striatal dopamine level. In addition, there was no significant difference in serum levels of creatinine, urea and potassium ions between the control and 6-OHDA treated groups.

Conclusion: Since the death of DA neurons in SNc is the main pathophysiological mechanism underlying in the development of both 6-OHDA induced Parkinsonism and $P D$ in human being, Cys $C$ and other markers of renal function cannot reflect DA neuronal death and accordingly cannot use for early diagnosis of PD.

\section{Keywords:}

Parkinson's disease;

6-hydroxydopamine;

Cystatin C;

Creatinine;

Urea;

Dopamine

\section{* Corresponding author: Hashem Haghdoost-Yazdi Email: \\ hhaghdoost@qums.ac.ir hhaghdoost@gmail.com Tel: +98 (28) 3366001-6}

Received 20 March 2019; Received in revised form 9 November 2019; Accepted 11 November 2019

http://dx.doi.org/10.32598/ppj.24.2.10

\section{Introduction}

Parkinson's disease (PD) is the second most common neurodegenerative disease after Alzheimer's disease affecting about 6 million people in the world (Lees et al., 2009). Since the age of human populations 
increases progressively, it is predicted that the prevalence of this disease increases twice in the next twenty years (Dorsey et al., 2007; Lees et al., 2009). The most important pathologic feature of $P D$ is the progressive death of the dopaminergic (DA) neurons in substantia nigra pars compacta (SNc). The mechanism of this neuronal death is unknown, but the mitochondrial dysfunction and oxidative stress play an important role in the events leading to the DA neuronal death (Jenner and Olanow, 1996; Dauer and Przedborski, 2003; Tsang and Chung, 2009; Moon and Paek, 2015).

Currently, PD is diagnosed by extrapyramidal signs such as tremor, bradykinesia and rigidity which appear after the death of more than $70 \%$ of DA neurons in SNc (Jankovic, 2008; Shulman et al., 2011). Therefore, one strategy for better management of $P D$ is the early recognition of DA neuronal death. The early diagnosis can significantly help us to choose the perfect treatment strategy and diminish or even reverse the neurodegenerative processes. One of the method for early detection of $P D$ is the identification of biomarkers for early detection of DA neuronal death in SNc. Nevertheless, identification of biomarker for $P D$ is still in the early stages and none of the introduced biomarkers were enrolled in clinical trials so far.

Cystatin C (CysC) is a 120-amino-acid protein produced by all cells in human being (Dharnidharka et al., 2002). This protein is a cysteine protease inhibitor that inhibits catepsines in vitro. A wide range of biological functions such as effect on growth progression, inhibition of inflammation and antimicrobial activity have been attributed to this protease (Lerner and Grubb, 1992; Tavera et al., 1992; Xiao et al., 2012). In human, CysC was first identified in the cerebrospinal fluid (CSF), but it has been shown that it is expressed by all mammalian cells and released in all body fluids (Bobek and Levine, 1992; Turk et al., 2008). CysC is freely filtered by glomerulus in the kidneys, metabolized in the primary tubule and is a sensitive indicator of renal function (Dharnidharka et al., 2002). It is also a powerful predictor of heart diseases such as heart failure and coronary artery disease (Angelidis et al., 2013). Since it is largely expressed by various cells and tissues in brain, it is possible that Cys C plays important role in CNS. Some studies have shown that CysC plays a protective role in neurodegenerative diseases (Xu et al., 2005; Gauthier et al., 2011; Yang et al., 2015; Mathews and Levy, 2016; Zou et al., 2017). On the other hand, there are evidences indicating that CysC induces neuronal death in the human CNS neurons and also in neurons of the granular layer of dentate gyrus in rat (Nagai et al., 2005). Also, an association between stroke and CysC was shown (Pirttilä and Pitkänen, 2006; Ni et al., 2007; Xiao et al., 2012). In animal models, the level of Cys $C$ in the hippocampus increases after an ischemic injury (Palm et al., 1995; Pirttilä and Pitkänen, 2006). On the other hand, CysC concentration reduces in the cerebrospinal fluid in patients with Alzheimer's disease (Hansson et al., 2009). There are considerable controversies about the role of Cys $\mathrm{C}$ in PD. It showed that no significant difference in CSF level of CysC between patients with PD and the agematched control (Přikrylová Vranová et al., 2010 and Yamamoto-Watanabe et al., 2010). On the other hand, several authors have shown that the serum level of CysC increases in these patients (Chen et al., 2015; Hu et al., 2016).

However, epidemiological and human studies have several limitations. They cannot clarify whether the association between Cys $\mathrm{C}$ and $\mathrm{PD}$ is merely related to a pre-existing high level of this substance in plasma or progressive DA neuronal death produces dynamic changes in the level of endogenous CysC. In addition, pharmacological therapy and comorbidity can increase this confliction. Therefore, in current study we examined the changes in serum level of CysC in the 6-hydroxydopamine (6-OHDA) rat model. In this model, although the behavioral symptoms are different from the clinical signs of $P D$ in human, but DA neuronal death occurs in SNc and accordingly the level of striatal dopamine decreases. Therefore, any association between the 6-OHDA induced Parkinsonism and serum level of Cys $C$ can introduce this substance as an indicator of DA neuronal death in SNc and even a biomarker for early diagnosis of PD. Also, since CysC is an indicator of renal function (Dharnidharka et al., 2002), the serum levels of creatinine, urea and potassium ions which are other markers of renal function were assessed to determine if these markers associate with DA neuronal death in SNc.

\section{Materials and methods}

Animals and experimental groups 
Twenty eight adult male Wistar rats (Razi Institute, Karaj, Iran), weighing 250-300g were housed in large cages $(38 \times 59 \times 20 \mathrm{~cm})$ at a temperature-controlled colony room under $12 \mathrm{~h}$ light/12h dark cycle with free access to tap water and standard food. All procedures carried out throughout this study were accordance with the guidelines of animal experiments of the Research Council at Qazvin University of Medical Sciences (ethical number: IR.QUMS.REC. 1394.312). Rats were divided into two experimental groups of control (con) which were not administered anything and 6-OHDA group, which intracerebrally received 6-OHDA. Each group consisted of 14 rats.

\section{Materials}

Thd 6-OHDA, apomorphine hydrochloride, Hamilton syringe, ascorbic acid, Triton X-100 and normal goat serum were purchased from Sigma-Aldrich Co. (St. Louis, MO). Anti-tyrosine hydroxylase (TH) antibody and fluorescently labeled rabbit secondary antibodies were purchased from Abcam Co. (Cambridge, United Kingdom). Mounting medium was purchased from Santa Cruz Co. (Heidelberg, Germany), xylazine and ketamine were purchased from Alfasan Co. (Nederland). ELISA kits for measurement of CysC and dopamine were purchased from Hangzhou Eastbiopharm (USA) and LDN (Nordhorn, Germany) companies. Other chemicals were purchased from Merck Co. (Germany).

\section{Surgical procedures}

The 6-OHDA was microinjected into medial forebrain bundle (MFB) of the right hemisphere through a $10 \mu \mathrm{l}$ Hamilton syringe and using stereotaxic surgery. Briefly, rats were anesthetized with intraperitoneally (ip) injection of a solution containing ketamine $(100 \mathrm{mg} / \mathrm{kg})$ and xylazine $(5 \mathrm{mg} / \mathrm{kg})$. Subsequently, $4 \mu \mathrm{l}$ of isotonic $\mathrm{NaCl}$ solution containing $0.2 \%$ of ascorbic acid and 6-OHDA $(4 \mu \mathrm{g} / \mu \mathrm{l})$ was microinjected into two sites at the following coordinates: anterior-posterior (AP): -4 , lateral (L): -1.8 , dorso-ventral (DV): 9 and AP: -4.4 , L: -2 , DV: 8.8. AP and $L$ were measured from bregma, while DV was measured from the surface of skull according to the atlas of Paxinos and Watson 2007.

\section{Apomorphine-induced rotational test}

Apomorphine induced rotational test was carried out before the toxin injection and in the third and fifth weeks after that (Fig. 1) according to the method we previously described (Haghdoost-Yazdi et al., 2010, Sarukhani et al., 2018). Briefly, animals were initially allowed to acclimate for a 5-min followed by an ip injection of apomorphine hydrochloride dissolved in saline $(0.5 \mathrm{mg} / \mathrm{kg})$. One minute later, an observer was directly counted the number of full rotations for $30 \mathrm{~min}$ in a cylindrical container (diameter: $28 \mathrm{~cm}$; height: $38 \mathrm{~cm}$ ). Contralateral (away from the injection side) and ipsilateral rotations were

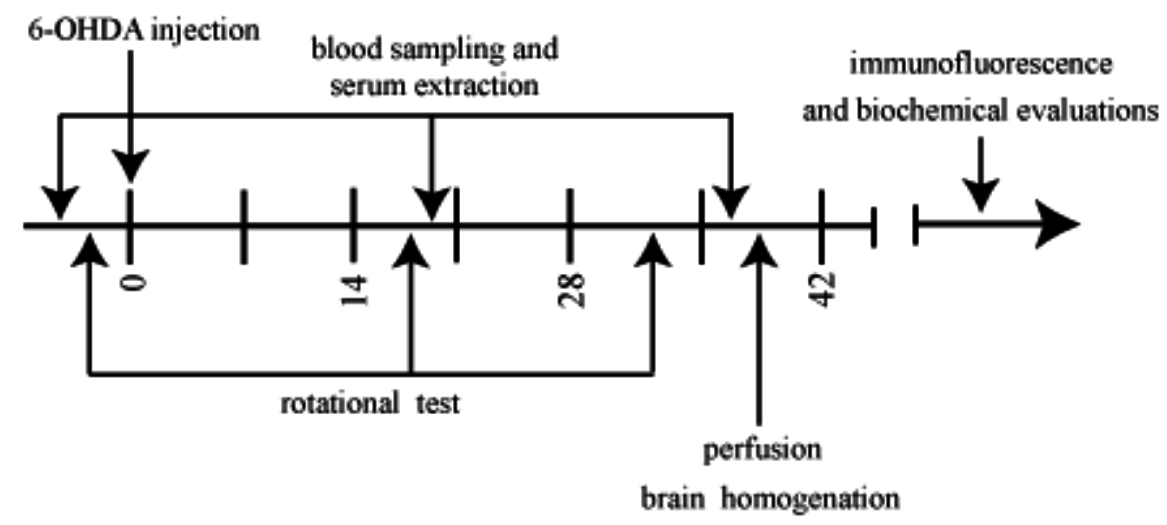

Fig.1. Experiment design. Only rats showed less than 10 rotations per hour in presurgery apomorphine- induced rotational test were used for experiments. Rotational test was also carried out at the third and fifth weeks after the 6- OHDA injection. Blood sampling and serum extractions were carried out at three times: before the toxin and at third and sixth weeks after that. In sixth week, six rats in each group were perfused and immunofluorescence staining was carried out on the midbrain sections. The brain of the other rats was freshly removed and separate homogenates were prepared from striatums of both hemispheres. Striatal dopamine level and CysC concentrations in serum were measured using immunosorbent assay kits. Numbers show the days after the toxin. 
counted as positive and negative scores, respectively, and the net number of rotations was defined as the positive scores minus the negative ones.

\section{Immunofluorescence experiment}

Immunofluorescence experiment was carried out on tissues of 6 rats from each group. After anesthesia using ketamine and xylazine, the rats were perfused transcardially with phosphate-buffered saline (PBS), followed by $4 \%$ paraformaldehyde. Subsequently, their brains were removed and postfixed overnight in paraformaldehyde and transferred to $30 \%$ sucrose in PBS at $4^{\circ} \mathrm{C}$. The midbrain section was isolated and frozen in a cryostat embedding medium (Bio- Optica, Italy) at $-22^{\circ} \mathrm{C}$. The coronal sections ( $8 \mu \mathrm{m}$ thickness) were prepared using a cryostat (Histo-Line Laboratories, Italy). One out of every serial 3 sections was permeabilized with $0.2 \%$ Triton $\mathrm{X}-100$ and blocked with $10 \%$ normal goat serum for 1 hour. The sections were incubated overnight at $4^{\circ} \mathrm{C}$ with anti-TH antibody (1:250) and incubated with proper fluorescently labeled rabbit secondary antibodies. Afterwards, the sections were coverslipped using a mounting medium, which contained 4',6-diamidino- 2phenylindole (DAPI) as the nuclear stain. They were then visualized on an Olympus microscope at $10 x$ magnification and sections including the SNc (AP, 4.8 to -5.2 relative to the bregma) were selected. Then five sections in each animal were selected from totally 50 sections which were divided into five series rostrocaudally. There were at least 5 -section intervals between 2 selected sections. TH-positive cells were visualized and counted manually using a light microscope at $400 \times$ magnification.

\section{Blood sampling and determination of CysC, creatinine, urea and $\mathrm{K}+$ concentrations in serum}

Blood sampling were performed at three time points: before the toxin injection and three as well as six weeks after that (Fig. 1). The first and second samples were collected from caudal vein and the third sample was collected from the heart of animals under the deep anesthesia. Blood was allowed to coagulate and then serum was separated and kept in $-80^{\circ} \mathrm{C}$ until to measurement time. Cys C was measured using specific ELISA kit based on its manufacturer's instruction. Creatinine, urea and $\mathrm{K}_{+}$ concentrations were measured using Jaffe calorimetric, urease and ion selective methods, respectively.

\section{Dopamine measurement}

Dopamine concentrations in the right and left striatums were measured in eight rats in each group using Dopamine Research ELISATM kit (BA E-5300, Nordhorn, Germany) according to manufacturer's instructions. Briefly, striatal tissues were homogenized in $\mathrm{HCl}(0.01 \mathrm{~N}, 1 \mathrm{ml}$ for $50 \mathrm{mg}$ tissue) with EDTA ( $1 \mathrm{mM})$ and sodium metabisulfite $(4 \mathrm{mM})$. In this condition, dopamine is charged positively and its solubility reached optimized level. The homogenate was centrifuged at $15000 \mathrm{~g}$ at $40 \mathrm{C}$ for $15 \mathrm{~min}$ and the supernatant was collected for measuring. Measurements were performed in $20 \mu \mathrm{l}$ of standards and diluted samples using a microplate reader set at $450 \mathrm{~nm}$ and a reference wavelength between 620 and $650 \mathrm{~nm}$. The concentration of dopamine in the sample was calculated according to the six standards from 0 to 90ng. This ELISA kit provides a very sensitive (lower limits at $0.7 \mathrm{ng} / \mathrm{ml}$ ) approach for measurement of dopamine.

\section{Statistical analysis}

Data were expressed as the mean \pm SEM, in spite of the probable non- normality of the distribution of scores. Data were initially analyzed by the Kolmogorov-Smirnov test to define their normality. Then, data between the groups were analyzed by $\mathrm{t}$ Test. Within the groups, data were subjected to analysis of variance (one-way ANOVA) followed by Newman-Keuls test to obtain probable significant differences between the groups. Statistically significant differences were considered at a $\mathrm{P} \leq 0.05$.

\section{Results}

Plot $A$ in Figure 2 illustrates the findings of apomorphine-induced rotational test and plot $\mathrm{B}$ illustrates the serum level of CysC in the control and 6- OHDA treated groups. While the rats in control group showed no significant rotation, most of the 6-OHDA treated showed high number of asymmetric rotations contralateral to injection site. The number of rotations in the fifth week after the toxin was significantly higher than that in the third week $(P<0.001)$. On the other hand, there was no significant difference in serum $\mathrm{Cys} C$ level between the control and 6-OHDA treated groups. In the control 

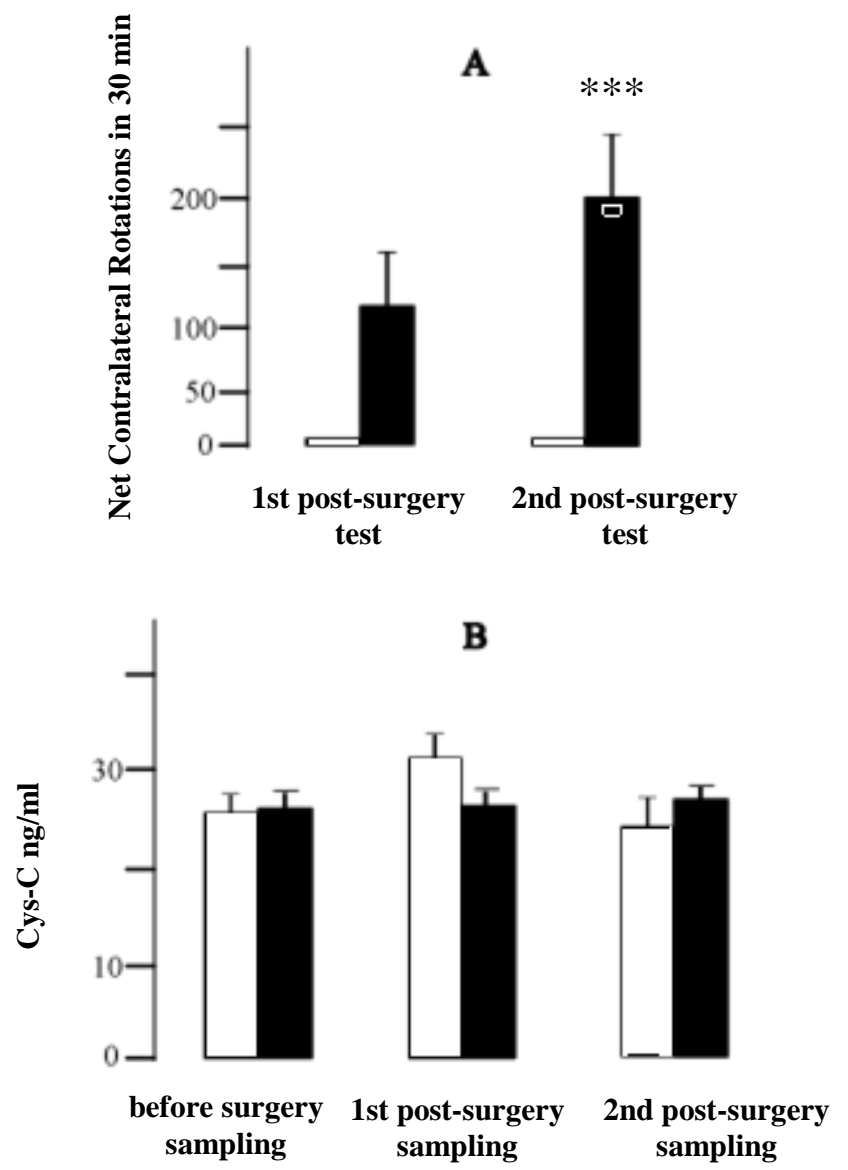

Fig.2. The 6-OHDA did not change the serum level of CysC. Plot A illustrates the net number of apomorphine-induced contralateral rotations in the third (1st post-surgery test) and fifth weeks (2nd post-surgery test) after the toxin. Asymmetric rotational behavior indicated the induction of Parkinsonism in rats. The number of rotations in the fifth week was much higher than that in the third week. Plot B illustrates the serum concentration of CysC before the toxin and in the third (1st post-surgery sampling) and sixth (2nd post-surgery sampling) weeks after that in the control (con, $n=14$ ) and 6-OHDA treated ( $n=14)$ rats. Administration of toxin and induction of Parkinsonism did not change significantly the serum level of $C y s C$. ${ }^{* * *} P<0.001$ compared to the number of rotations in the third week after the toxin.

group before the toxin and in the third and sixth weeks after that, CysC concentrations were $26.2 \pm 0.8,31 \pm 1.9$ and $25 \pm 2.1 \mathrm{ng} / \mathrm{ml}$, respectively. In the 6-OHDA group, these values were $26.6 \pm 0.8$, $27 \pm 0.7$ and $27.3 \pm 0.5 \mathrm{ng} / \mathrm{ml}$. Statistical analysis indicated no correlation between CysC and severity of apomorphine-induced rotations in 6-OHDA treated group (Pearson's correlation coefficient was 0.091, $P<0.55$, for third week after the toxin and 0.101 , $P<0.44$ for sixth week after the toxin).

A careful analysis of the rotational behavior showed that the intensity of rotational behavior was not the same among 6-OHDA treated rats and some of them showed high number of rotations, others showed low number of rotations and still others showed no significant rotations. For better examination, we divided 6-OHDA treated rats into two groups: severe group $(n=8)$ that showed more than 300 rotations per hour in one of the post-surgery rotational tests and mild group $(n=6)$ that showed less than 50 rotations per hour. Plot $A$ in Figure 3 illustrates the findings of apomorphine-induced rotational test and plot $\mathrm{B}$ illustrates the serum level of CysC in these groups. In the severe group and not in the mild group, the number of rotations in the fifth week was much higher than that in the third week $(P<0.001)$. On the other hand, there was no significant difference in the serum level of CysC between the severe and mild groups. Also, no significant correlation was found between the severity of rotations and the serum CysC level in these groups.

Cys $C$ is known as an indicator of renal disorders. In the current study, we also measured the serum concentrations of potassium, urea and creatinine, which increase as a result of renal malfunctions. Figure 4 illustrates the serum concentrations of these 


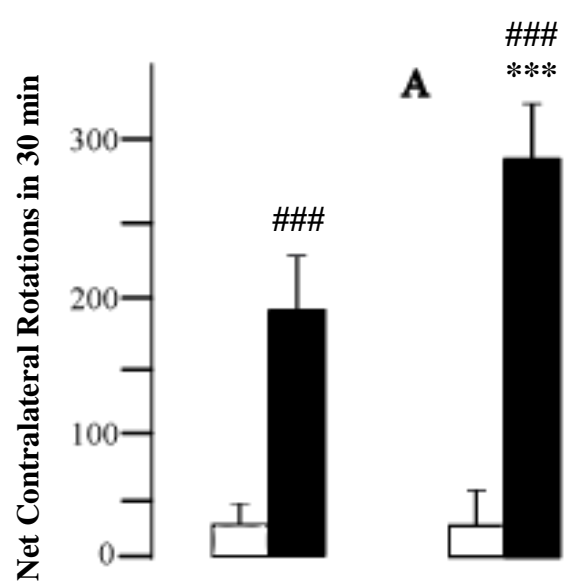

$\begin{array}{cc}\text { 1st post-surgery } & \text { 2nd post-surgery } \\ \text { test } & \text { test }\end{array}$

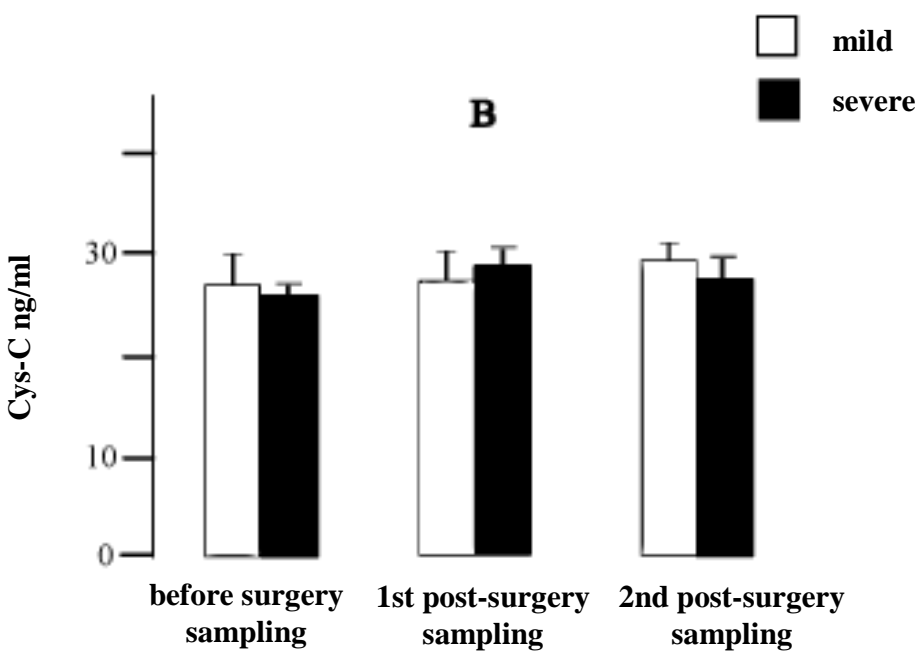

Fig.3. There was no significant difference in serum level of CysC between rats with severe and mild behavioral symptoms. Plot $A$ illustrates the number of apomorphine-induced rotations in the third and fifth weeks after the toxin in the severe (showed more than 300 rotations in one hour, $n=8$ ) and mild (showed less than 50 rotations in one hour, $n=6$ ) groups. In contrast to the mild group, the severity of behavioral symptoms in the severe group in the fifth week after the toxin was significantly higher than that in the third week. Plot B illustrates the serum level of CysC in the severe and mild groups before the toxin and in the third and sixth weeks after that. ${ }^{\# \# \#} P<0.001$ compared to the mild group; ${ }^{* \star} P<0.001$ compared to the number of rotations in the third week after the toxin.

substances in control and 6-OHDA treated groups (upper plots) and also in the severe and mild groups (lower plots). The concentration of potassium in the control and 6-OHDA treated groups was $6.07 \pm 0.4$ and $6.05 \pm 0.44 \mathrm{meq} / \mathrm{l}$, respectively. Concentrations of urea and creatinine in the control group were $64 \pm 2.9$ and $0.8 \pm 0.07 \mathrm{mg} / \mathrm{dl}$, respectively.

These values in the 6-OHDA treated group were $62 \pm 1.3$ and $0.71 \pm 0.03 \mathrm{mg} / \mathrm{dl}$. As you can see, there was no significant difference in the serum concentration of these substances between the control and 6-OHDA treated groups. Similar to CysC, there was no significant difference in the serum concentrations of these substances between the severe and mild groups. The only significant difference was the lower concentration of creatinine in the severe group compared to control group. Statistical analysis showed that there was no significant correlation between the severity of rotations and serum levels of creatinine, urea and potassium.

Figure 5 illustrates the survival rate of DA neurons (TH-positive) in the substantia nigra and also the concentration of dopamine in the striatum. As photomicrographs show, in severe 6-OHDA treated rats (Fig. 5B), the survival rate of $\mathrm{TH}$-positive neurons was very low and $70 \%$ of these neurons in the lesioned hemisphere lost (plot A). On the other hand, the survival rate of $\mathrm{TH}$-positive neurons was much higher in rats with mild number of rotations (Fig. 5C). 

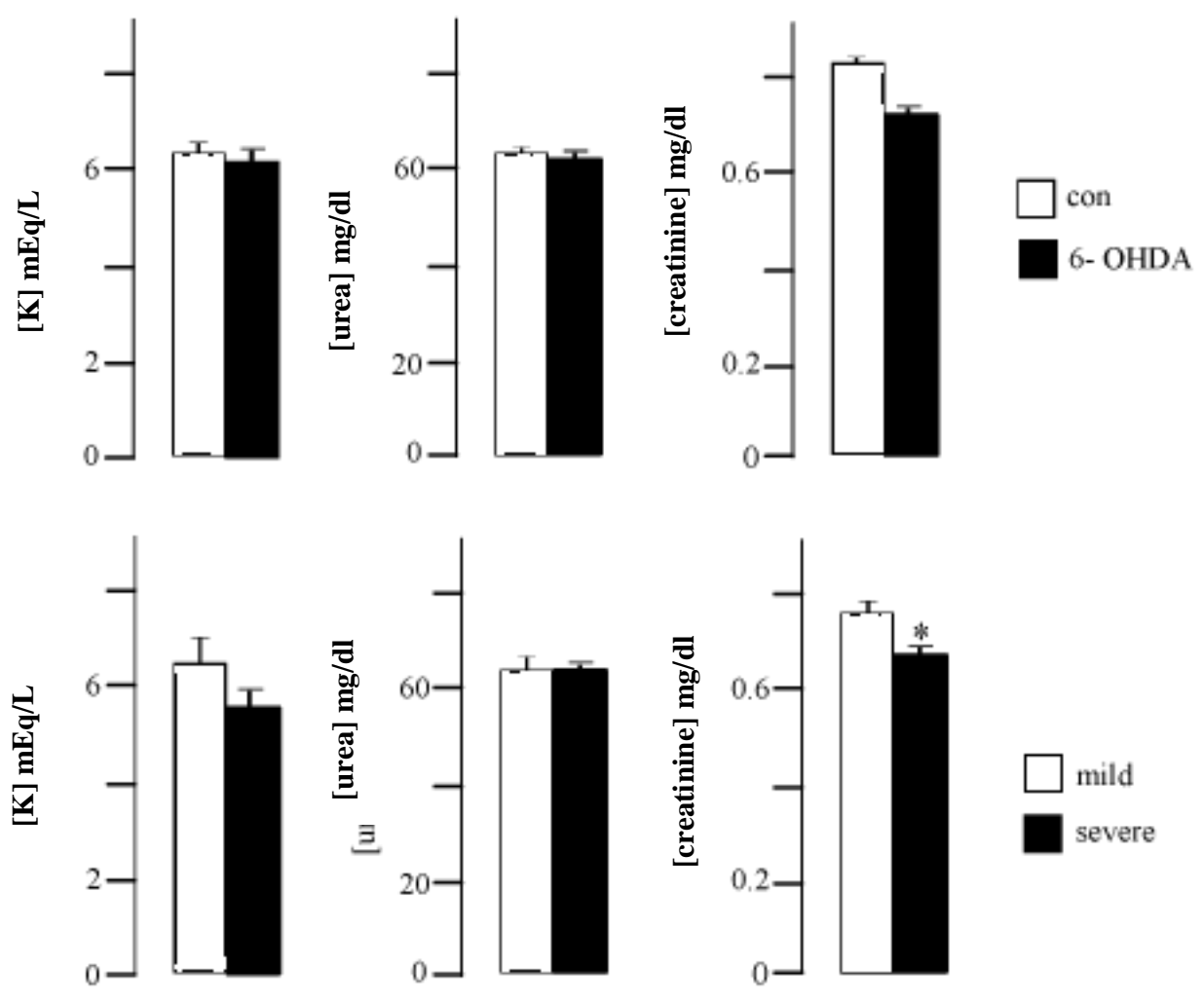

Fig.4. The 6-OHDA administration did not change significantly the serum levels of potassium, urea and creatinine. The upper plots illustrate the serum levels of these substances in the control (con) and 6-OHDA treated groups in the sixth week after the toxin. The lower plots illustrate the serum levels of these substances in severe and mild groups in this week. " $P<0.05$ compared to the control group.

In this group, only $40 \%$ of the neurons in the lesioned hemisphere lost. The values of striatal dopamine level also showed similar results and the level of dopamine in the lesioned hemisphere was $70 \%$ and $30 \%$ less than that in intact hemisphere in severe and mild groups, respectively (plot B). Statistical analysis showed there was no significant correlation between serum level of CysC and survival rate of DA neurons in $\mathrm{SNc}$ and also the striatal dopamine level (Pearson's Correlation coefficient $0.0588, P<0.45$ between serum CysC level and number of $\mathrm{TH}$ - positive neurons in $\mathrm{SNc}$ and $0.132, P<0.19$ between serum Cys $C$ level and right striatal dopamine level).

\section{Discussion}

In this study, we evaluated the association between serum levels of CysC and other markers of renal functions and the intensity of behavioral symptoms of 6-OHDA-induced Parkinsonism in rats. We also assessed survival rate of DA neurons in SNc and striatal dopamine level using immunofluorescence and biochemical techniques. Our data indicate that there is no association between serum levels of CysC, creatinine, urea and potassium ions, as well as the number of rotations in apomorphine-induced rotational test. Also, no association was observed between these substances and the DA neuronal death in SNc or striatal dopamine level.

Various studies showed that change in serum Cys $C$ level is associated with chronic renal disease, urinary tract infections, cancer, hypertension, cardiovascular disease, rheumatoid arthritis and aging (Bjornstad et al., 2015; Joshi and Viljoen, 2015; Taal, 2015). The concentration of CysC in CSF is four to five times higher than that in the serum (Wilson et al., 2013; Ren et al., 2015) indicating CysC may have important physiological and pathophysiological roles in the brain. In agreement with this, several studies have described an association between CysC and neurologic disorders (Simonsen et al., 2007; TsujiAkimoto et al., 2009; Haghdoost-Yazdi et al., 2010; Wilson et al., 2010). There are controversies regarding the role of this substance in PD. Some authors reported a positive correlation between PD and CysC (Přikrylová Vranová et al., 2010; Yamamoto-Watanabe et al., 2010) but others showed no significant association (Chen et al., 2015; Hu et al., 

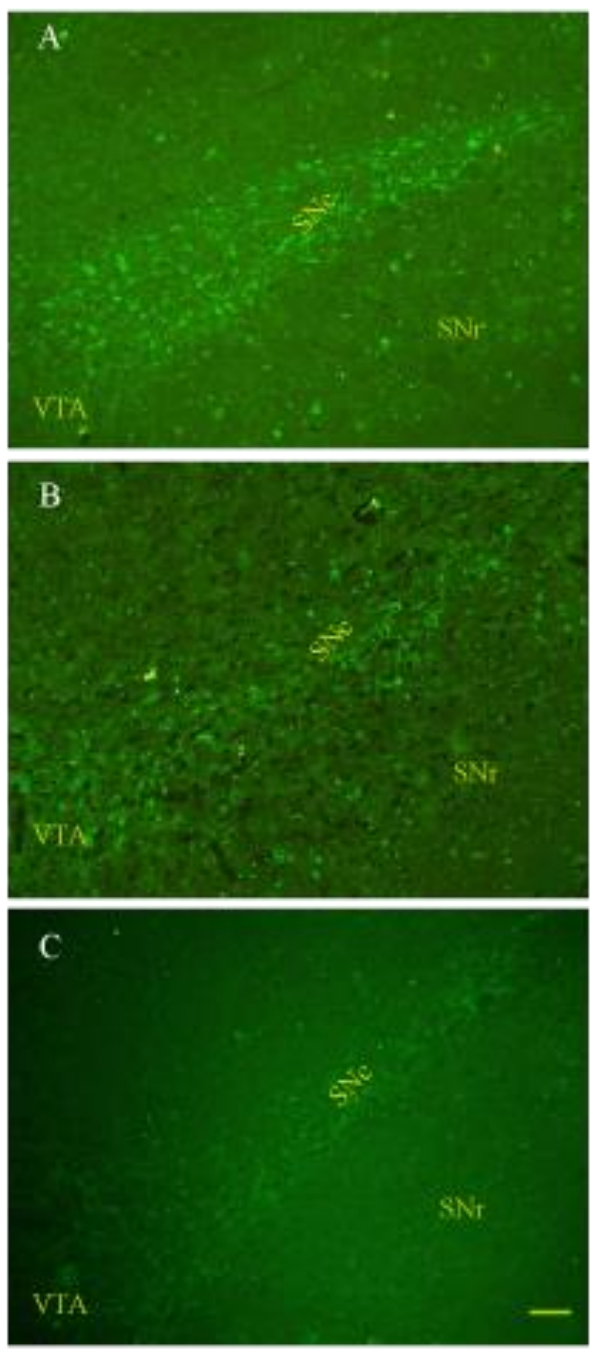
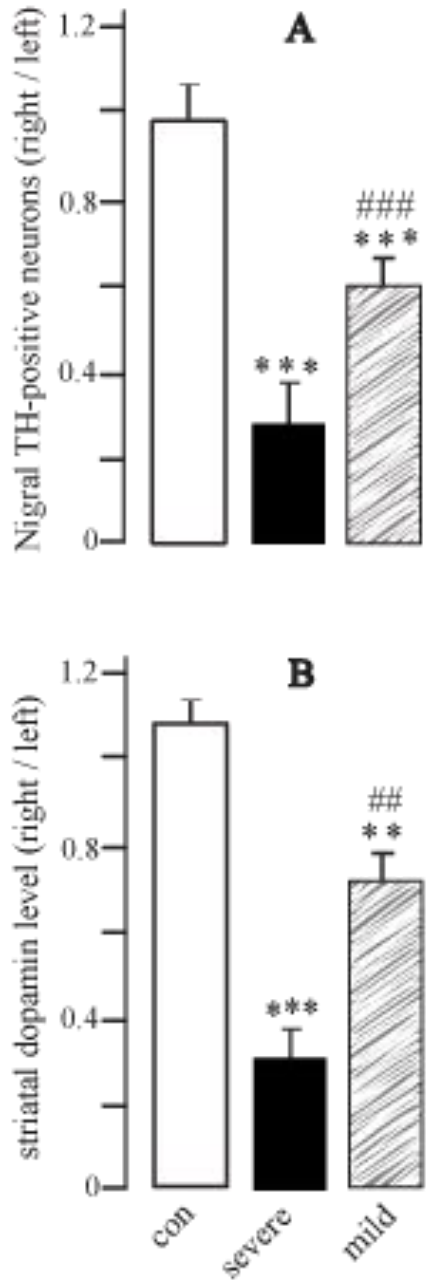

Fig.5. Photomicrographs show tyrosine hydroxylase (TH)-positive neurons in the substantia nigra of the lesioned hemisphere in the control (con, A), severe (B) and mild (C) groups. The survival rate of TH-positive neurons in the severe group was much less than that in the mild and control groups. Plot $\mathrm{A}$ illustrates the ratio of number of $\mathrm{TH}$-positive neurons in the lesioned hemisphere to the intact hemisphere in these groups. As it has been seen in mild group, although the survival rate was much higher than that in the severe group, there was still considerable neuronal death in compare to intact side. Plot B illustrates the ratio of striatal dopamine level in the lesioned hemisphere to the intact hemisphere in these groups. A distinct and significant difference is observed in the concentration of striatal dopamine in the lesioned hemispheres among the control, severe and mild groups. SNc: substantia nigra pars compacta, SNr: substantia nigra pars reticulate, VTA: ventral tegmental area. ${ }^{* \star *} P<0.001$ and ${ }^{* \star} P<0.01$ compared to the control group or intact hemisphere. ${ }^{\# \# \#} P<0.001$ and ${ }^{\# \#} P<0.01$ compared to the severe group.

2016). Animal studies evaluated more carefully this association. $\mathrm{Xu}$ and her colleagues showed that in rats two weeks after the injection of 6-OHDA into the MFB region, the number of $\mathrm{Cys} C$ immunoreactive cells increases in striatum and substantia nigra (Xu et al., 2005). In agreement with this findings, studies on PC12 cells. showed that 6-OHDA increases expression and release of CysC in these cells (Lee et al., 2006). This increase in expression of CysC immunoreactive cells is in line with the pattern of DA neurodegeneration in SNc after the injection of 6-
OHDA into MFB region. In acute model of 6-OHDA induced Parkinsonism, the intensity of DA neuronal death in SNc in the fifth week after the toxin is higher than that in the second or third weeks (Sarukhani et al., 2018). According this, in current study behavioral tests were performed in the third and fifth weeks after the toxin and the serum CysC level was measured before and in the third and sixth weeks after the toxin. Along with the reported neuronal death pattern, our findings showed that the number of rotations significantly increased in function of time and in the 
fifth week after the toxin was significantly higher than that in the third week. However, our data shows that the serum level of $\mathrm{Cys} C$ did not change after the toxin and no correlation found between this level and intensity of behavioral symptoms.

Injection of the 6-OHDA into the MFB region results in the rapid and extensive degeneration of DA neurons in SNc. Rotational behavior reflects the degree of DA neuronal death in SNc (Shimohama et al., 2003; Yuan et al., 2005; Sarookhani et al., 2018). However, the association between DA neuronal death and severity of rotational behavior is not perfect and it has been reported that this behavior can only distinct partial and intensive neuronal death in SNc, and cannot distinguish moderate death levels. Also, it has been reported that only rats with severe (>90\%) DA neuronal death show significant rotational behavior (Yuan et al., 2005). Therefore, for more examination of the association between the serum level of Cys $C$ and the DA neuronal death, we examined the survival rate of these neurons in SNc. Also, as PD in humans, 6-OHDA induced Parkinsonism is associated with degeneration of DA terminals and decreased levels of dopamine in the striatum. Therefore, the level of striatal dopamine was also measured. Immunohistofluorescence examination showed the extensive DA neuronal loss in the lesioned hemisphere of 6-OHDA treated rats. The degree of this neuronal loss in rats with severe behavioral symptoms was much higher than in that in rats with mild symptoms. In line with this, striatal dopamine level in 6-OHDA treated rats was much less than that in the control group. As DA neuronal survival rate, there was a roughly negative correlation between striatal dopamine levels with severity of behavioral symptoms. Therefore, our findings showed that the severity of rotational behavior reflects somehow the degree of DA neuronal loss and reduced level of striatal dopamine. Accordingly, our data suggest that the serum level of Cys $C$ cannot predict or reflect the intensity of DA neuronal death of DA in SNc.

Evidences show that in PD in addition to dopaminergic system, other neurotransmitter systems, such as the noradrenergic and serotoninergic systems, are also damaged (Lindgren et al., 2014). Disorders in these systems, especially the noradrenergic system, can produce autonomic disorders such as constipation (Zhang et al., 2015). There are also reports describing an association between PD and renal disorders and uremia (Ali et al., 1985; Lin et al., 2016). Our previous study showed that 6-OHDA induced Parkinsonism associates with the reduction in serum urate level (Sarukhani et al., 2018). Therefore, administration of 6-OHDA in the brain can cause changes in other parts of the body and serum. Since CysC is an indicator of renal disorders, we also measured the serum levels of creatinine, urea and potassium ions, which are other indicators of the renal function. It was reported that the creatinine concentration in CSF of patients with $P D$ is lower than that of the control subjects (Agren and Niklasson, 1988; Garseth et al., 2000; Trupp et al., 2014). On the other hand, Lu et al. (2014) showed that the creatinine level in the brain of gold fish treated with MPTP (1-methyl-4-phenyl1,2,3,6-tetrahydropyridine), a toxin similar to 6-OHDA that causes Parkinsonism in mice and primates, is significantly higher than that in the control group. Our findings showed that there is no significant difference in serum creatinine level between the 6-OHDA and control groups. There was also no significant difference between the severe and mild groups, but in the severe group, creatinine level was significantly lower than that in the control group. As we described before, severe Parkinsonian rats showed severe DA neuronal death in SNc. Therefore, even if lower creatinine level indicate severe degeneration of DA neurons, it cannot consider as a biomarker for PD, because the clinical symptoms are also appeared at this level of neuronal death. On the other hand, decrease in creatinine level may indicate a change in energy metabolism (Agren and Niklasson, 1988). It has been reported that in patients with PD, the CSF levels of alanine, lactic acid and glucose are reduced, indicating that the glucose metabolism is affected in this disease (Öhman and Forsgren, 2015).

Our findings showed that serum levels of urea and potassium do not change in 6-OHDA induced. Also, the levels of these two substances did not associate with the severity of behavioral symptoms, the degree of DA neuronal death in SNc and striatal dopamine level. The important point is that a significant change in the serum concentration of potassium ions can cause the death by inducing cardiac arrhythmias. Therefore, only the survival of rats can indicate the lack of significant changes in potassium ion concentration. However, this change could be small and has no physiological or pathophysiological 
demonstrations, but has a diagnostic value. On the other hand, small changes in the concentration may not reach a statistically significant level. In this study, the samples were collected from 14 rats in each group that allowed even small changes in serum potassium, as well as urea or creatinine, reach to the significant level. Therefore, given that the 6-OHDA injection and the resulting neuronal death did not change the serum concentration of Cys $\mathrm{C}$, creatinine, urea and potassium, it can be said that 6-OHDA induced Parkinsonism is not associate with renal disorders. Therefore, the indicators of renal disorders are not suitable for the early diagnosis of PD.

The main limitation of this study was the inability to measure $\mathrm{CysC}$, creatinine, urea and potassium ions in CSF of rats. It is possible that the level of these substances in the CSF was a more precise index for 6-OHDA induced Parkinsonism. However, we could not measure the level of CysC in CSF of rats because its volume is too small and there are several technical problems in sampling. However, it has been shown that there is an association between the CSF level of Cys $\mathrm{C}$ and its level in serum. Another limitation was the small amount of blood sample that can be collected from the tail of conscious rat. Regarding to the very small sera volumes, only the CysC level were measured in samples collected from the tails and the other three substances were only measured in the samples collected from the heart.

\section{Conclusion}

Our data indicate that there is no association between serum levels of CysC, creatinine, urea and potassium ions, and 6-OHDA-induced Parkinsonism in rats. Although the behavioral symptoms of $6-\mathrm{OHDA}$ induced Parkinsonism are significantly different from the clinical signs of PD, the death of the DA neurons and the reduction in striatal dopamine level are common in both. Therefore, our data suggests that CysC is not involve in pathophysiology of PD. Also, the data shows that DA neuronal cell death does not change serum level of CysC or produce renal malfunctions demonstrating they cannot be considered as biomarkers for the early diagnosis of PD.

\section{Acknowledgments}

We would like to thank Mrs. Ayda Faraji for her assistance in stereotaxic surgeries and Ms. Zare for her contribution to immunohistofluorescence experiments. This study was supported by a grant-inaid for scientific research from the Research Council of Qazvin University of Medical Sciences.

\section{Conflict of interest}

The authors declare no conflicts of interest.

\section{References}

Ali F, Tayeb O, Attallah A. Plasma and brain catecholamines in experimental uremia: acute and chronic studies. Life Sci 1985; 37: 1757-64. https://doi.org/10.1016/0024-3205(85)90217-6

Agren $\mathrm{H}$, Niklasson F. Creatinine and creatine in CSF: indices of brain energy metabolism in depression. Short note. J Neural Transm 1988;74: 55-9. https://doi.org/ 10.1007/BF01243575

Angelidis C, Deftereos S, Giannopoulos G, Anatoliotakis N, Bouras G, Hatzis $G$, et al. Cystatin C: an emerging biomarker in cardiovascular disease. Curr Top Med Chem 2013; 13: 164-79. https://doi.org/10.2174/15680 26611313020006

Bjornstad P, Cherney DZ, Maahs DM, Nadeau KJ. Fat mass is associated with Cystatin $\mathrm{C}$ and estimated glomerular filtration rate in adolescents with type 1 diabetes. J Ren Nutr 2015; 25: 454-5. https://doi.org/ 10.1053/j.jrn.2015.04.007

Bobek LA, Levine MJ. Cystatins--inhibitors of cysteine proteinases. Crit Rev Oral Biol Med 1992; 3: 307-32. https://doi.org/10.1177/10454411920030040101

Chen WW, Cheng X, Zhang X, Zhang QS, Sun HQ, Huang WJ, et al. The expression features of serum Cystatin $C$ and homocysteine of Parkinson's disease with mild cognitive dysfunction. Eur Rev Med Pharmacol Sci 2015; 19(16):2957-63.

Dauer W, Przedborski S. Parkinson's disease: mechanisms and models. Neuron 2003; 39: 889-909. https://doi.org/10.1016/S0896-6273(03)00568-3

Dharnidharka VR, Kwon C, Stevens G. Serum cystatin C is superior to serum creatinine as a marker of kidney function: a meta-analysis. Am J Kidney Dis 2002; 40: 221-6. https://doi.org/10.1053/ajkd.2002.34487

Dorsey ER, Constantinescu R, Thompson JP, Biglan KM, Holloway RG, Kieburtz K, et al. Projected number of people with Parkinson disease in the most populous nations, 2005 through 2030. Neurology; 2007; 68: 3846. https://doi.org/10.1212/01.wnl.0000247740.47667.03

Garseth M, Sonnewald U, White LR, Rod M, Zwart JA, Nygaard $O$, et al. Proton magnetic resonance spectroscopy of cerebrospinal fluid in neurodegenerative disease: indication of glial energy impairment in Huntington chorea, but not Parkinson disease. J Neurosci Res 2000; 60: 779-82. https://doi.org/10.1002/1097-4547(20000615)60:6<779 ::AID-JNR10>3.0.CO;2-M

Gauthier S, Kaur G, Mi W, Tizon B, Levy E. Protective mechanisms by cystatin $C$ in neurodegenerative 
diseases. Front Biosci (Schol Ed) 2011; 3: 541-54. https://doi.org/10.2741/s170

Haghdoost-Yazdi H, Hosseini SS, Faraji A, Nahid D, Jahanihashemi $H$. Long term exposure to norharman exacerbates 6-hydroxydopamine-induced parkinsonism: possible involvement of L-type Ca2+ channels. Behav Brain Res 2010; 215: 136-40. https://doi.org/10.1016/ j.bbr.2010.07.011

Hansson SF, Andreasson U, Wall M, Skoog I, Andreasen N, Wallin A, et al. 2009. Reduced levels of amyloidbeta-binding proteins in cerebrospinal fluid from Alzheimer's disease patients. J Alzheimers Dis 2009; 16: 389-97. https://doi.org/10.3233/JAD-2009-0966

Hu WD, Chen J, Mao CJ, Feng P, Yang YP, Luo WF, et al. Elevated Cystatin $C$ levels are associated with cognitive impairment and progression of parkinson disease. Cogn Behav Neurol 2016; 29: 144-9. https://doi.org/10.1097/ WNN.0000000000000100

Jankovic J. Parkinson's disease: clinical features and diagnosis. J Neurol Neurosurg Psychiatry 2008; 79: 368-76. https://doi.org/10.1136/jnnp.2007.131045

Jenner $\mathrm{P}$, Olanow CW. Oxidative stress and the pathogenesis of Parkinson's disease. Neurology 1996; 47: S161-70. https://doi.org/10.1212/WNL.47.6_Suppl 3. $161 \mathrm{~S}$

Joshi S, Viljoen A. Renal biomarkers for the prediction of cardiovascular disease. Curr Opin Cardiol 2015; 30: 454-60. https://doi.org/10.1097/HCO.0000000000000 177

Lee DC, Close FT, Goodman CB, Jackson IM, WightMason C, Wells LM, et al. Enhanced cystatin C and lysosomal protease expression following 6hydroxydopamine exposure. Neurotoxicology 2006; 27: 260-76. https://doi.org/10.1016/j.neuro.2005.11.011

Lees AJ, Hardy J, Revesz T. Parkinson's disease. Lancet 2009; 373: 2055-66. https://doi.org/10.1016/S01406736(09)60492-X

Lerner UH, Grubb A. Human cystatin C, a cysteine proteinase inhibitor, inhibits bone resorption in vitro stimulated by parathyroid hormone and parathyroid hormone-related peptide of malignancy. J Bone Miner Res 1992; 7: 433-40. https://doi.org/10.1002/jbmr. 5650070411

Lin SY, Lin CL, Hsu WH, Yeh HC, Lin CC, Lin CH, et al. Association between acute kidney injury and risk of Parkinson disease. Eur J Intern Med 2016; 36: 81-86. https://doi.org/10.1016/j.ejim.2016.09.016

Lindgren HS, Demirbugen $M$, Bergqvist $F$, Lane $E L$, Dunnett SB. The effect of additional noradrenergic and serotonergic depletion on a lateralised choice reaction time task in rats with nigral 6-OHDA lesions. Exp Neurol 2014; 253: 52-62. https://doi.org/10.1016/j.expneurol. 2013.11.015

Lu Z, Wang J, Li M, Liu Q, Wei D, Yang M, Kong L. (1)H NMR-based metabolomics study on a goldfish model of Parkinson's disease induced by 1-methyl-4-phenyl1,2,3,6-tetrahydropyridine (MPTP). Chem Biol Interact 2014; 223:18-26. https://doi.org/10.1016/j.cbi.2014.09. 006
Mathews PM, Levy E. Cystatin $C$ in aging and in Alzheimer's disease. Ageing Res Rev 2016; 32: 38-50. https://doi.org/10.1016/j.arr.2016.06.003

Moon HE, Paek SH. Mitochondrial dysfunction in Parkinson's disease. Exp Neurobiol 2015; 24: 103-16. https://doi.org/10.5607/en.2015.24.2.103

Nagai A, Ryu JK, Terashima M, Tanigawa Y, Wakabayashi K, McLarnon JG, et al. Neuronal cell death induced by cystatin $\mathrm{C}$ in vivo and in cultured human CNS neurons is inhibited with cathepsin B. Brain Res 2005; 1066: 120-8. https://doi.org/10.1016/j.brainres.2005.10.063

Ni L, Lu J, Hou LB, Yan JT, Fan Q, Hui R, et al. Cystatin C, associated with hemorrhagic and ischemic stroke, is a strong predictor of the risk of cardiovascular events and death in Chinese. Stroke 2007; 38: 3287-8. https://doi.org/10.1161/STROKEAHA.107.489625

Öhman A, Forsgren L. NMR metabonomics of cerebrospinal fluid distinguishes between Parkinson's disease and controls. Neurosci Lett 2015; 594: 36-9. https://doi.org/10.1016/j.neulet.2015.03.051

Palm DE, Knuckey NW, Primiano MJ, Spangenberger AG, Johanson CE. Cystatin C, a protease inhibitor, in degenerating rat hippocampal neurons following transient forebrain ischemia. Brain Res 1995; 691: 1-8. https://doi.org/10.1016/0006-8993(95)00520-Z

Paxinos G, Watson C. The rat brain in stereotaxic coordinates. 6th ed. San Diego, CA: Academic Press; 2007

Pirttilä TJ, Pitkänen A. Cystatin C expression is increased in the hippocampus following photothrombotic stroke in rat. Neurosci Lett 2006; 395: 108-13. https://doi.org/ 10.1016/j.neulet.2005.10.091

Přikrylová Vranová $H$, Mareš J, Nevrlý $M$, Stejskal D, Zapletalova J, Hluštík $P$, et al. CSF markers of neurodegeneration in Parkinson's disease. J Neural Transm (Vienna) 2010; 117: 1177-81. https://doi.org/ 10.1007/s00702-010-0462-z

Ren Y, Zhu W, Cui F, Yang F, Chen Z, Ling L, et al. Measurement of cystatin $C$ levels in the cerebrospinal fluid of patients with amyotrophic lateral sclerosis. Int $\mathrm{J}$ Clin Exp Pathol 2015; 8: 5419-26.

Sarookhani MR, Haghdoost-Yazdi $H$, Sarbazi-Golezari A, Babayan-Tazehkand A, Rastgoo N. Involvement of adenosine triphosphate-sensitive potassium channels in the neuroprotective activity of hydrogen sulfide in the 6hydroxydopamine-induced animal model of Parkinson's disease. Behav Pharmacol 2018; 29: 336-343. https://doi.org/10.1097/FBP.0000000000000358

Sarukhani MR, Haghdoost-Yazdi H, Khandan-Chelarci G. Changes in the serum urate level can predict the development of Parkinsonism in the 6hydroxydopamine animal model. Neurochem Res 2018; 43: 1086-1095. https://doi.org/10.1007/s11064-0182522-y

Sarukhani M, Haghdoost-Yazdi $H$, Sarbazi Golezari A, Babayan-Tazehkand A, Dargahi T, Rastgoo N. Evaluation of the antiparkinsonism and neuroprotective effects of hydrogen sulfide in acute 6-hydroxydopamineinduced animal model of Parkinson's disease: 
behavioral, histological and biochemical studies. Neurol Res 2018; 40: 523-531. https://doi.org/10.1080/ 01616412.2017 .1390903

Shimohama S, Sawada H, Kitamura Y, Taniguchi T. Disease model: Parkinson's disease. Trends Mol Med 2003; 9: 360-5. https://doi.org/10.1016/S1471-4914(03) 00117-5

Shulman JM, De Jager PL, Feany MB. Parkinson's disease: genetics and pathogenesis. Annu Rev Pathol 2011; 6: 193-222. https://doi.org/10.1146/annurevpathol-011110-130242

Simonsen AH, McGuire J, Podust VN, Hagnelius NO, Nilsson TK, Kapaki E, et al. A novel panel of cerebrospinal fluid biomarkers for the differential diagnosis of Alzheimer's disease versus normal aging and frontotemporal dementia. Dement Geriatr Cogn Disord 2007; 24: 434-40. https://doi.org/10.1159/ 000110576

Taal MW. Chronic kidney disease in older people diagnosis, aetiology and consequences. Curr Opin Nephrol Hypertens 2015; 24 :475-9. https://doi.org/10. 1097/MNH.0000000000000164

Tavera C, Leung-Tack J, Prevot D, Gensac MC, Martinez J, Fulcrand $\mathrm{P}$, et al. Cystatin $\mathrm{C}$ secretion by rat glomerular mesangial cells: autocrine loop for in vitro growthpromoting activity. Biochem Biophys Res Commun 1992;182:1082-8.https://doi.org/10.1016/0006-291X(92) 91842-E

Trupp M, Jonsson P, Ohrfelt A, Zetterberg H, Obudulu O, Malm L, et al. Metabolite and peptide levels in plasma and CSF differentiating healthy controls from patients with newly diagnosed Parkinson's disease. J Parkinsons Dis 2014; 4: 549-60. https://doi.org/10. 3233/JPD-140389

Tsang $\mathrm{AH}$, Chung KK. Oxidative and nitrosative stress in Parkinson's disease. Biochim Biophys Acta 2009; 1792: 643-50. https://doi.org/10.1016/j.bbadis.2008.12.006

Tsuji-Akimoto S, Yabe I, Niino M, Kikuchi S, Sasaki H. Cystatin $\mathrm{C}$ in cerebrospinal fluid as a biomarker of ALS. Neurosci Lett 2009; 452: 52-5. https://doi.org/10.1016/ j.neulet.2009.01.026

Turk V, Stoka V, Turk D. Cystatins: biochemical and structural properties, and medical relevance. Front Biosci 2008; 13: 5406-20. https://doi.org/10.2741/3089

Wilson ME, Boumaza I, Bowser R. Measurement of cystatin
C functional activity in the cerebrospinal fluid of amyotrophic lateral sclerosis and control subjects. Fluids Barriers CNS. 2013; 10: 15. https://doi.org/10. 1186/2045-8118-10-15

Wilson ME, Boumaza I, Lacomis D, Bowser R. Cystatin C: a candidate biomarker for amyotrophic lateral sclerosis. PLoS One. 2010; 5: e15133. https://doi.org/10.1371/ journal.pone.0015133

Xiao D, Liu H, Zhang H, Luo Y. Impact of cystatin C levels on infarct size and hemorrhage volume in acute cerebral stroke. J Neurol 2012; 259: 2053-9. https://doi.org/10.1007/s00415-012-6453-2

Xu L, Sheng J, Tang Z, Wu X, Yu Y, Guo H, et al. Cystatin $C$ prevents degeneration of rat nigral dopaminergic neurons: in vitro and in vivo studies. Neurobiol Dis 2005; 18: 152-65. https://doi.org/10.1016/j.nbd.2004.08. 012

Yamamoto-Watanabe Y, Watanabe M, Jackson M, Akimoto $\mathrm{H}$, Sugimoto $\mathrm{K}$, Yasujima $\mathrm{M}$, et al. Quantification of cystatin $C$ in cerebrospinal fluid from various neurological disorders and correlation with G73A polymorphism in CST3. Brain Res 2010; 1361: 140-5. https://doi.org/10.1016/j.brainres.2010.09.033

Yang B, Zhu J, Miao Z, Zhou B, Ge W, Zhao H, et al. Cystatin $\mathrm{C}$ is an independent risk factor and therapeutic target for acute ischemic stroke. Neurotox Res 2015; 28: 1-7. https://doi.org/10.1007/s12640-015-9522-3

Yuan H, Sarre S, Ebinger G, Michotte Y. Histological, behavioural and neurochemical evaluation of medial forebrain bundle and striatal 6-OHDA lesions as rat models of Parkinson's disease. J Neurosci Methods 2005; 144: 35-45. https://doi.org/10.1016/j.jneumeth. 2004.10.004

Zhang X, Li Y, Liu C, Fan R, Wang P, Zheng L, et al. Alteration of enteric monoamines with monoamine receptors and colonic dysmotility in 6hydroxydopamine-induced Parkinson's disease rats. Transl Res 2015; 166: 152-62. https://doi.org/10.1016/ j.trsl.2015.02.003

Zou J, Chen Z, Wei X, Chen Z, Fu Y, Yang X, et al. Cystatin $C$ as a potential therapeutic mediator against Parkinson's disease via VEGF-induced angiogenesis and enhanced neuronal autophagy in neurovascular units. Cell Death Dis 2017; 8: e2854. https://doi.org/ $10.1038 /$ cddis.2017.240 